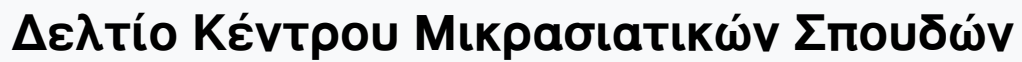

Tóp. 6 (1986)

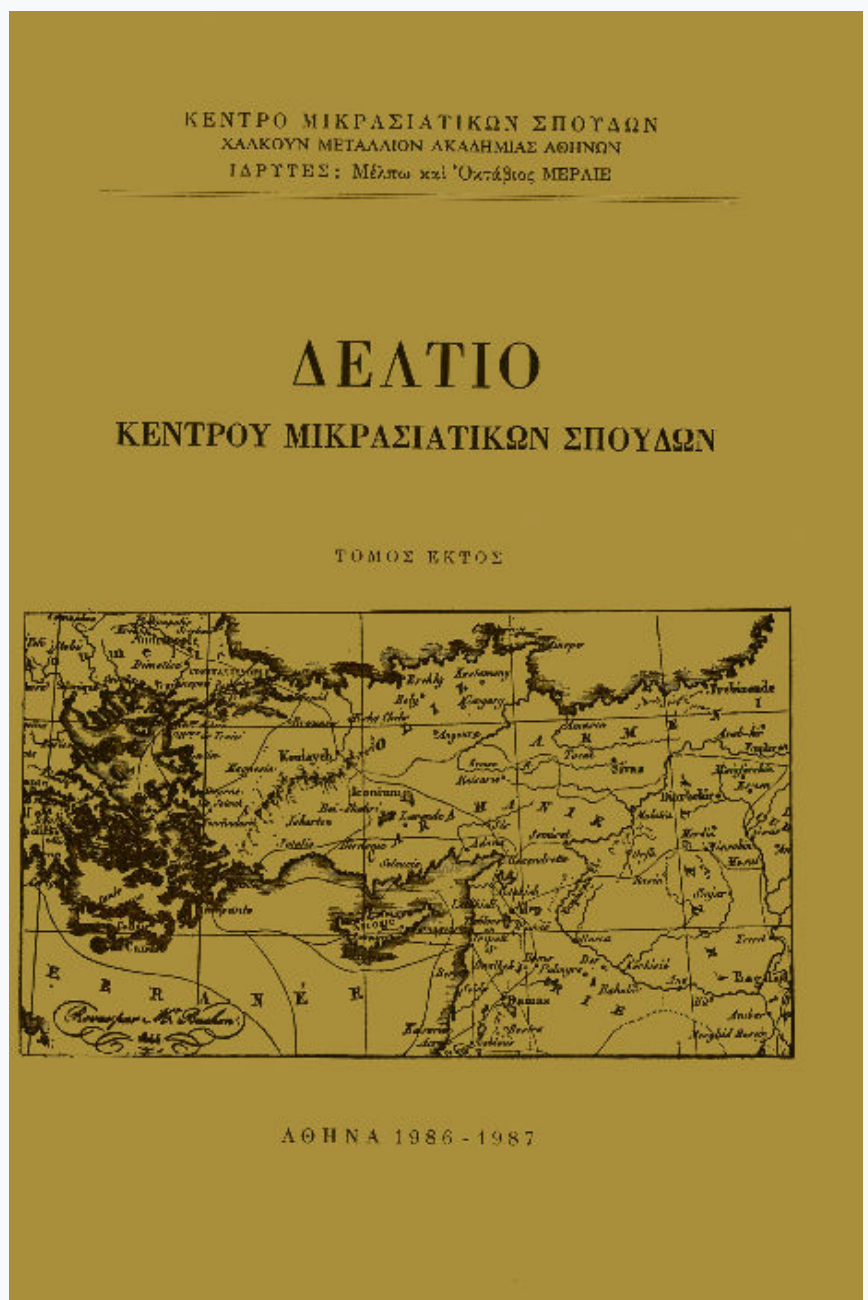

Ethnic survival, nationalism and forced migration

Paschalis M. Kitromilides, Alexis Alexandris

doi: $\underline{10.12681 / \text { deltiokms.116 }}$

Copyright $(2015$, Paschalis M. Kitromilides, Alexis Alexandris

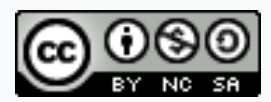

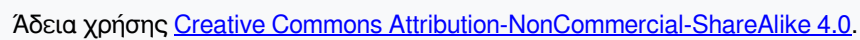

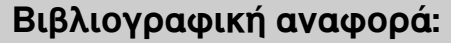

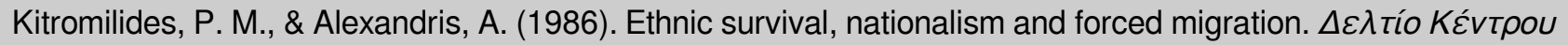

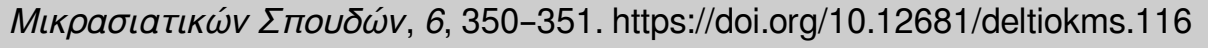




\section{ETHNIC SURVIVAL, NATIONALISM AND FORCED MIGRATION}

\section{Corrigendum}

A proofreading error has caused an incongruence in the data presented in column $\varepsilon^{\prime}$ of Table VII (Appendix B) in our article ${ }^{1}$ on the historical demography of the Greek community of Asia Minor at the close of the Ottoman era in volume V of the Deltio (p. 44). The table presented basic statistics on the Greek schools of the Krini diocese in the Çeşme peninsula of Western Asia Minor across the channel from the Greek island of Chios. We reproduce therefore the table in its entirety, with the correct figures in column $\varepsilon^{\prime}$.

PASCHALIS M. KITROMILIDES - ALEXIS ALEXANDRIS

1. Paschalis M. Kitromilides - Alexis Alexandris, «Ethnic Survival, Nationalism and Forced Migration. The Historical Demography of the Greek Community of Asia Minor at the

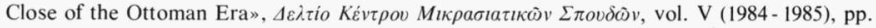
9-44. 
APPENDIX B

TABLE VII

GREEK SCHOOLS OF THE KRINI DIOCESE*

\begin{tabular}{|c|c|c|c|c|c|c|c|c|}
\hline & $a^{\prime}$ & $\beta^{\prime}$ & $\gamma^{\prime}$ & $\delta^{\prime}$ & $\varepsilon^{\prime}$ & $\sigma \tau^{\circ}$ & $\zeta$ & $\eta$ \\
\hline 1. Krini (Çeșme) & Cessme & 1 & 1 & 8 & 8 & 456 & 360 & $560 \mathrm{TL}$ \\
\hline 2. Alaçata & $"$ & 1 & 1 & 7 & 8 & 414 & 279 & $450 \mathrm{TL}$ \\
\hline 3. Kato Panagia & " & 1 & 1 & 3 & 4 & 253 & 206 & $240 \mathrm{TL}$ \\
\hline 4. Agia Paraskevi & 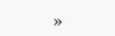 & $i$ & 1 & 2 & 3 & 160 & 124 & $180 \mathrm{TL}$ \\
\hline 5. Ovacik & $"$ & Mixed & - & 2 & - & 40 & 22 & $30 \mathrm{TL}$ \\
\hline 6. Reisdere & " & 1 & 1 & 2 & 2 & 110 & 90 & $57 \mathrm{TL}$ \\
\hline 7. Kerme Yalısı & " & Mixed & - & 1 & - & 20 & 10 & $10 \mathrm{TL}$ \\
\hline 8. Aghrelia & $"$ & Mixed & - & 1 & - & 35 & 16 & $15 \mathrm{TL}$ \\
\hline 9. Pyrghi & $"$ & Mixed & - & 1 & - & 25 & 10 & $10 \mathrm{TL}$ \\
\hline 10. Erythrai & " & 1 & 1 & 2 & 1 & 86 & 75 & $75 \mathrm{TL}$ \\
\hline 11. Çığköy & " & Mixed & - & 1 & - & 10 & 8 & $8 \mathrm{TL}$ \\
\hline 12. Ahırlı & Karaburun & 1 & 1 & 1 & 1 & 50 & 30 & $60 \mathrm{TL}$ \\
\hline 13. Sahibi & " & 1 & 1 & 1 & 1 & 40 & 60 & $70 \mathrm{TL}$ \\
\hline 14. Ambar-Seki & 》 & Mixed & - & 1 & - & 10 & 5 & $8 \mathrm{TL}$ \\
\hline 15. Mikro Mourdouvani & $"$ & 1 & 1 & 1 & 1 & 60 & 30 & $61 \mathrm{TL}$ \\
\hline 16. Tekkes & " & 1 & 1 & 1 & 1 & 50 & 40 & $50 \mathrm{TL}$ \\
\hline $\begin{array}{l}\text { 17. Mega Mourdouvani } \\
\text { (Inçepinar) }\end{array}$ & ” & 1 & 1 & 2 & 1 & 90 & 100 & $80 \mathrm{TL}$ \\
\hline 18. Monastırı & " & Mixed & - & 1 & - & 30 & 6 & $20 \mathrm{TL}$ \\
\hline 19. Tepepoz & " & Mixed & - & 1 & - & 50 & 25 & $20 \mathrm{TL}$ \\
\hline 20. Yeni Liman & $"$ & Mixed & - & 1 & - & 50 & 25 & $20 \mathrm{TL}$ \\
\hline 21. Kaş-Seki & $"$ & Mixed & - & 1 & - & 25 & 7 & $10 \mathrm{TL}$ \\
\hline 22. Şarbıncık & " & Mixed & - & 1 & - & 20 & 10 & $10 \mathrm{TL}$ \\
\hline 23. Sancak & " & Mixed & - & 1 & - & 50 & 10 & $20 \mathrm{TL}$ \\
\hline 24. Boynak & " & Mixed & - & 1 & - & 85 & 20 & $30 \mathrm{TL}$ \\
\hline 25. Salman & $"$ & Mixed & - & 1 & - & 20 & 10 & $20 \mathrm{TL}$ \\
\hline 26. Egri Liman & $"$ & Mixed & - & 1 & - & 10 & 5 & $10 \mathrm{TL}$ \\
\hline 27. Deniz Giren & $"$ & Mixed & - & 1 & - & 17 & 19 & $26 \mathrm{TL}$ \\
\hline 28. Küçük Bahçe & $"$ & Mixed & - & 1 & - & 15 & 15 & $8 \mathrm{TL}$ \\
\hline 29. Meli & $»$ & Mixed & - & 2 & - & 130 & 80 & $80 \mathrm{TL}$ \\
\hline 30. Glezonisi & Vurla & Mixed & - & 2 & - & 85 & 25 & $35 \mathrm{TL}$ \\
\hline 31. Kioseni & $"$ & - & - & - & - & - & - & \\
\hline 32. Agia Paraskevi & $"$ & Mixed & - & 1 & - & 15 & 10 & $10 \mathrm{TL}$ \\
\hline TOTAL & & 31 & 11 & 53 & 31 & 2.511 & 1.732 & $2.283 \mathrm{TL}$ \\
\hline
\end{tabular}

* $\alpha^{\prime}$ : Name of Kaza. $\beta^{\prime}$ : Number of boy's schools. $\gamma^{\prime}:$ Number of girls's schools. $\delta^{\prime}$ : Male teachers. $\varepsilon^{\prime}$ : Female teachers. $\sigma \tau^{\prime}$ : Number of boy students. $\zeta^{\prime}:$ Number of girl students. $\eta^{\prime}$ : School expenditure during academic year 1909-1910 in Turkish lira (TL). 